\title{
Manejo quirúrgico de cáncer papilar de tiroides con invasión traqueal: Reporte de dos casos en Servicio de Otorrinolaringología de Hospital Carlos Van Buren
}

\author{
Surgical treatment in papillary thyroid carcinoma with tracheal invasion. \\ Report of two cases in Otolaryngology Service of Carlos Van Buren Hospital
}

\author{
José Ignacio Rojas B. ${ }^{1}$, Francisca Fernández A. ${ }^{2,3}$
}

\section{Resumen}

La invasión traqueal corresponde al tercer sitio más común de extensión extratiroidea del cáncer de tiroides. Se debe sospechar en caso de disnea, disfonía, estridor y hemoptisis, sin embargo, hasta un $30 \%$ de los pacientes se pueden presentar de forma asintomática, pesquisándose al momento de la cirugía. La nasofibroscopía de rutina, una alta sospecha y una planificación quirúrgica adecuada, serán fundamentales para lograr una resección tumoral completa y mejorar tanto el pronóstico como el control local. A continuación, presentamos dos casos clínicos manejados con resección cricotraqueal con anastomosis término-terminal en el Servicio de Otorrinolaringología del Hospital Carlos Van Buren.

Palabras clave: cáncer tiroides, invasión traqueal, resección traqueal.

\begin{abstract}
Tracheal invasion is the third most common site of extrathyroideal invasion of thyroid cancer, recognized as a por prognostic indicator. It should be suspected in case of dyspnea, dysphonia, stridor, and hemoptysis. However, up to $30 \%$ of cases are asymptomatic, detected at the time of surgery. Routine nasofibroscopy, thorough examination and surgical planning are essential to ensure a complete tumoral resection, improvement in prognostic and local control of disease. We present two cases with cricotracheal resection and end-to-end anastomosis in Otholaringology Service from the Carlos Van Buren Hospital.

Keywords: thyroid cancer, tracheal invasion, tracheal resection.
\end{abstract}

\author{
'Servicio de Otorrinolaringología, \\ Hospital Carlos Van Buren, \\ Universidad de Valparaíso. \\ Valparaíso, Chile. \\ 2Departamento de Especialidades, \\ Cátedra de Otorrinolaringología, \\ Escuela de Medicina, Facultad \\ de Medicina, Universidad de \\ Valparaíso. Viña del Mar, Chile. \\ ${ }^{3}$ Servicio de Otorrinolaringología, \\ Hospital Carlos Van Buren. \\ Valparaíso, Chile. \\ Los autores declaran no tener \\ conflictos de intereses. \\ Recibido el 17 de junio de 2020. \\ Aceptado el 16 de agosto de \\ 2020. \\ Correspondencia: \\ José Ignacio Rojas B. \\ 3 Poniente 220, Depto. 1102 \\ Viña del Mar, Chile. \\ Email: josrojasb@udd.cl
}

\section{Introducción}

El cáncer de tiroides es la neoplasia endocrina más frecuente, corresponde al 3\% de todos los tumores malignos. En términos histológicos, podemos subdividirlos en diferenciados, medulares y anaplásicos ${ }^{1}$. Los tumores diferenciados (carcinoma papilar, carcinoma folicular y de células de Hurthle) corresponden al 95\% de los casos y se originan en las células foliculares de la glándula tiroides ${ }^{2}$. Esta enfermedad puede evolucionar como un continuo, desde un carcinoma papilar hacia variantes de peor pronóstico como el de células altas, insular y luego pobremente diferenciado, para finalmente convertirse en un tumor anaplásico ${ }^{1}$, con tasas de supervivencia por sobre el $98 \%$ en carcinomas papilares y tasas de mortalidad por sobre el $98 \%$ en el caso de tumores anaplásicos ${ }^{3}$. Son factores de mal pronóstico edad $>45$ años, extensión extratiroidea, tamaño $>4$ $\mathrm{cm}$, pobre diferenciación histológica y metástasis a distancia ${ }^{4}$. La incidencia de extensión extratiroidea en cánceres bien diferenciados fluctúa entre un $6 \%$ a $13 \% \%^{5,6}$, siendo el sitio más común la musculatura infrahioidea (53\%) $\mathrm{y}$ en segundo lugar el nervio laríngeo recurrente $(47 \%)^{7}$. Menos frecuente es la invasión 
del tracto aerodigestivo (tráquea, esófago, laringe $y / o$ hipofaringe), con una incidencia entre el 1\%-8\%, los cuales se suelen presentar con histologías pobremente diferenciadas o anaplásicas $(60 \%-70 \%)^{8}$, siendo también más común en pacientes mayores, tumores de larga evolución o más periféricos y posteriores ${ }^{9}$. Si bien la extensión muscular puede ser fácilmente resecada sin mayor impacto en el pronóstico, la extensión posterior puede ser un reto quirúrgico, con alto riesgo de recurrencia local.

La invasión traqueal corresponde al tercer sitio más común de extensión extratiroidea, correspondiente al 37\% de los casos de extensión extratiroidea ${ }^{7}$. Los síntomas más comunes son disnea, disfonía, hemoptisis y estridor, siendo este último el más frecuente (aproximadamente un tercio de los pacientes). Otros elementos sugerentes de invasión traqueal son el compromiso cutáneo, disfagia y aspiración ${ }^{11}$. Sin embargo, entre un $12,5 \%$ a $30 \%$ de los pacientes pueden presentarse de forma asintomática y ser pesquisados durante el intraoperatorio ${ }^{10}$. Por lo tanto, es recomendable realizar nasofibroscopía prequirúrgica a todos los pacientes y ampliar el estudio con traqueoscopía e imágenes en caso de sospecha. La tomografía computarizada (TC) será de utilidad para determinar el compromiso de cartílago traqueal o cricoideo, la extensión local y compromiso de linfonodos cervicales y paratraqueales, siendo superior al estudio con ultrasonido en este contexto ${ }^{13}$.

La biopsia por punción con aguja fina (PAAF) podrá eventualmente confirmar el diagnóstico de malignidad y en casos de citologías seleccionadas, advertirnos de la agresividad $^{11}$. Sin embargo, es necesario considerar la frecuente heterogeneidad intratumoral, lo cual tiene relación con su comportamiento biológico, eventos oncogénicos y curso clínico. Rara vez el carcinoma papilar existe como un tumor homogéneo microscópicamente y esta coexistencia de variantes histopatológicas pueden ser clínicamente relevantes si una de las variantes representa un subtipo más agresivo como puede ser, la variante de células altas, células columnares y sólida ${ }^{14}$, otorgando un desafío adicional durante la sospecha diagnóstica. Por otro lado, la biopsia core se ha sugerido como una alternativa en caso de resultado no diagnóstico de la PAAF, sospecha de linfoma o cáncer anaplásico ${ }^{13}$.

Si bien todos los pacientes con compromiso traqueal se etapifican como T4a según la clasificación $\mathrm{TNM}^{15}$, veremos que engloba todo un espectro de formas clínicas. Para poder orientarnos durante el manejo será de utilidad el sistema de etapificación de Shin y cols. (Figura 1), siendo el más utilizado en la literatura,

Figura 1. Sistema de etapificación de invasión traqueal (adaptación realizada por Dra. Francisca Fernández A. de Shin y cols. $\left.{ }^{16}\right)$.

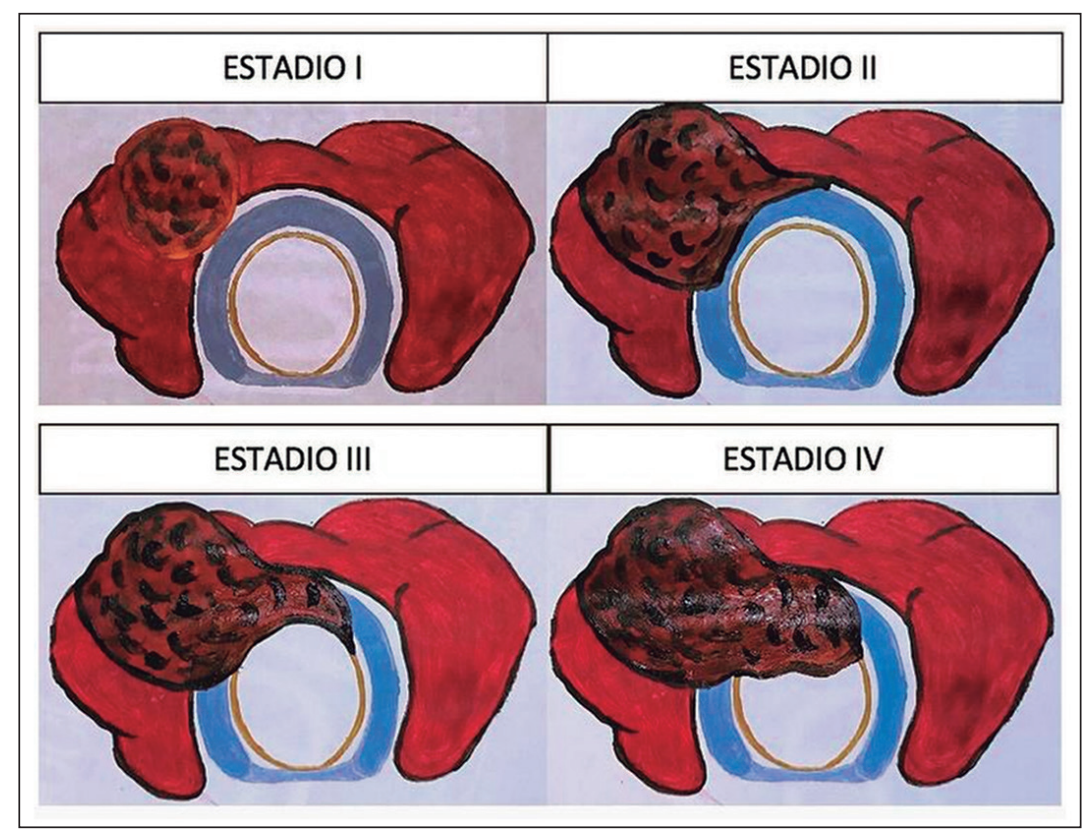


basado en la profundidad de la invasión ${ }^{16}$. Otra clasificación es la de Czaja y McCaffrey ${ }^{17}$, sin embargo, esta última engloba en conjunto la invasión traqueal y laringofaríngea. La invasión laríngea debe analizarse como una entidad diferente, donde el shaving, laringectomía parcial y laringectomía total deben considerarse como posibilidades quirúrgicas.

El tratamiento quirúrgico con márgenes negativos es el gold standard en el manejo de tumores bien diferenciados ${ }^{18}$. La escisión tangencial del tumor o resección tipo shaving, será exclusiva para estadios I; la resección en ventana de todo el espesor en caso de compromiso de un solo anillo traqueal o menos de un tercio de la circunferencia; resección circunferencial segmentaria traqueal o cricotraqueal con anastomosis término-terminal en los casos más avanzados ${ }^{19}$. Además, se debe tener en cuenta las comorbilidades del paciente, el grado de extensión de la enfermedad y la morbilidad secundaria a la cirugía. Finalmente, en caso de tumores indiferenciados debemos considerar alternativas de carácter paliativo.

En cuanto a su evolución, Nishida y cols. describen el manejo de 54 pacientes con antecedentes de tumores diferenciados en Etapa II o superior de acuerdo a la clasificación de Shin, quienes fueron sometidos a resección de vía aérea o resección subtotal sin resección de vía aérea, demostrando altas tasas de recurrencia local (79\% contra $8 \%$ ) y disminución de supervivencia global ( 1,5 contra 8,7 años) en el grupo de resección subtotal, objetivando la importancia de lograr una resección tumoral completa. Por otro lado, los pacientes en estadio I que fueron sometidos a procedimientos tipo shave, no tuvieron diferencias significativas en recurrencia local y regional, metástasis a distancia o sobrevida global, al compararlos con pacientes sin compromiso traqueal ${ }^{20}$. Sin embargo, aquelos pacientes sometidos a procedimientos tipo shave que desarrollan recurrencia, tendrían una peor sobrevida libre de enfermedad y sobrevida global en comparación con aquellos pacientes que si se les realiza resecciones traqueales desde un inicio ${ }^{18}$. Distinto es el caso de tumores anaplásicos que invaden las estructuras vecinas en etapas tempranas de su enfermedad, con tasas de sobrevida entre 3 y 6 meses, siendo el compromiso de vía aérea junto a la enfermedad metastásica, las causas de muerte más común ${ }^{21}$.
A continuación, se describen dos casos clínicos manejados con resección cricotraqueal o traqueal con anastomosis término-terminal, en el Servicio de Otorrinolaringología del Hospital Carlos Van Buren.

\section{Caso Clínico 1}

Paciente de sexo femenino de 61 años, sin antecedentes mórbidos, hábito tabáquico suspendido (IPA: 4), con historia de disnea progresiva de 2 años de evolución, manejada inicialmente como asma bronquial. Evoluciona con rápido deterioro, presentando disnea de reposo, estridor, disfonía y disfagia lógica, por lo que decide consultar en servicio de urgencia. Al examen físico, ingresa hemodinámicamente estable, sin requerimientos de oxígeno, pero con estridor evidente y disnea de reposo. A la nasofibroscopía presenta tumor exofítico, friable, que ocluye el lumen traqueal, con movilidad cordal conservada. $\mathrm{Al}$ TAC de cuello con contraste, se observa lesión de aspecto tumoral de origen tiroideo, del lóbulo izquierdo e istmo de $31 \times 26 \mathrm{~mm}$, con invasión y obstrucción de lumen traqueal de un $80 \%$ (y $20 \mathrm{~mm}$ de extensión) y adenopatías de aspecto metastásico en grupos II y III izquierdos. La PAAF informó un carcinoma papilar de tiroides. Con estos antecedentes se planifica resección traqueal segmentaria con anastomosis término-terminal. En el intraoperatorio destaca invasión del nervio recurrente izquierdo pese a la ausencia de parálisis cordal. Además, se observa extensión tumoral del borde inferior de cricoides y 4 anillos traqueales hacia caudal. Se decide realizar resección cricotraqueal con anastomosis término-terminal y disección cervical, conservando uno de los nervios recurrentes. Se mantiene con intubación orotraqueal en posoperatorio inmediato, evolucionando con atelectasias bibasales, pero con buen control gasométrico y hemodinámico. Es extubada a las 96 horas en pabellón bajo visión endoscópica. Dada de alta once días poscirugía. Biopsia confirma cáncer papilar de tiroides con extensión transmural a pared traqueal con bordes negativos. Tiroglobulina posquirúrgica negativa, actualmente 6 meses poscirugía sin signos sugerentes de recidiva, en espera de radioyodo. 


\section{Caso Clínico 2}

Paciente de sexo femenino de 64 años con antecedente de cáncer papilar de tiroides operada de tiroidectomía total con shaving traqueal más $200 \mathrm{mCi}$ de radioyodo. Dos años más tarde presenta recidiva local, realizándose resección cricotraqueal en ventana de la pared anterolateral derecha, con reconstrucción con cartílago costal de la pared lateral y traqueostoma (Figura 2), cursando con un accidente cerebrovascular (ACV) posquirúrgico inmediato no secuelado. Se indica nueva dosis de radioyodo con un cintigrama posradioyodo no captante.

Se presenta en comité oncológico para radioterapia, la cual se rechaza por antecedente de ACV. Durante evolución es decanulada según protocolo, destacando nódulo paratraqueal derecho de crecimiento progresivo, sin embargo paciente rechaza nuevas cirugías. Cuatro años después, consulta por disnea progresiva y estridor, sin otro síntoma asociado. A la nasofibroscopía destaca inmovilidad de cuerda vocal derecha conocida y ocupación traqueal por tumor exofítico y friable. Al TC de cuello con contraste se observa ocupación endotraqueal por tumor exofítico que ocluye alrededor de un 70\%. Considerando el antecedente previo de carcinoma papilar, se decide resección traqueal circunferencial de 2 anillos traqueales de extensión incluyendo parte del injerto costal y anastomosis término-terminal.

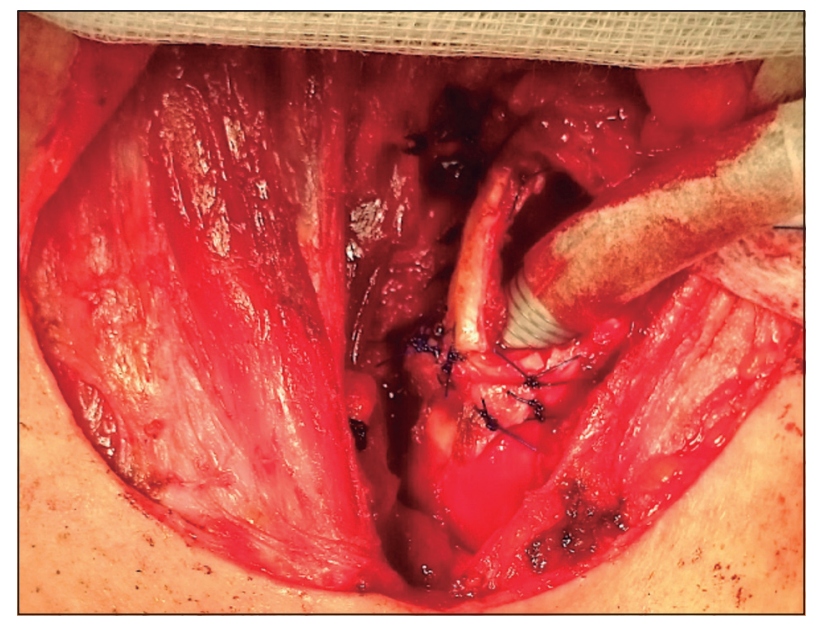

Figura 2. Resección en ventana y reconstrucción de la pared lateral traqueal más anastomosis.
Se mantiene intubada en posoperatorio inmediato y es extubada en pabellón a las 72 horas bajo visión endoscópica. Tres días posterior a extubación evoluciona con estridor inspiratorio y aumento de requerimientos de oxígeno, por lo que es reintubada bajo visión directa. Al estudio destaca atelectasias bibasales con foco de condensación. Finalmente, evoluciona con respuesta favorable a tratamiento antibiótico y soporte ventilatorio, siendo nuevamente extubada a los 8 días sin inconvenientes. Dada de alta 29 días poscirugía. Biopsia confirma carcinoma papilar con infiltración transmural de pared traqueal. Se presenta caso en comité oncológico y se decide solo seguimiento, sin indicación de radioyodo por antecedentes de tumor no radiocaptante y dosis previas de $400 \mathrm{mCi}$ de radioyodo. Actualmente 6 meses poscirugía, sin signos sugerentes de recidiva.

\section{Discusión}

La primera resección traqueal con reconstrucción descrita en humanos fue realizada por Dr. Grillo en la década de los 60, donde realizó distintas alternativas para reparar lesiones traqueales extensas. La técnica quirúrgica originalmente fue aplicada para el manejo de estenosis subglótica-traqueal y tumores primarios, siendo posteriormente utilizado para carcinomas con invasión traqueal. Su principal aporte fue demostrar que la liberación traqueal amplia, en conjunto con suturas libres de tensión y bordes sanos, permite resecar grandes porciones traqueales. El objetivo final es la resección quirúrgica completa sin enfermedad residual macroscópica ${ }^{22}$.

En ambos casos clínicos se decidió realizar una resección segmentaria (cricotraqueal y traqueal) con anastomosis término-terminal de acuerdo a los lineamientos descritos en la literatura ${ }^{23}$, logrando la resección completa del tumor. Con respecto al estudio preoperatorio, dada la necesidad de intervención quirúrgica para asegurar la vía aérea en ambos casos, no se complementó con biopsias core o quirúrgicas previas. Del mismo modo, se desestimó una traqueostomía quirúrgica y biopsia en primer tiempo quirúrgico dado que le agregaría complejidad a la resección tumoral subsiguiente. Este tipo de resección 
no sólo permite el control local, sino también el aumento de la supervivencia y alivio de los síntomas en tumores localmente extensos. Si bien, se puede optar a realizar cirugías menos agresivas, la presencia de tumor residual llevará a recurrencia y cirugías de revisión aún más difíciles. La resección circunferencial segmentaria y anastomosis término-terminal permite el estudio meticuloso de los márgenes y una reconstrucción inmediata de la vía aérea, siendo parte de la clave del éxito la sutura de los cabos traqueales sin tensión y la preservación de la funcionalidad de la laringe. En caso de parálisis de cuerda vocal, ya sea por iatrogenia, compromiso tumoral o necesidad de resección, se pueden plantear diferentes opciones terapéuticas, incluida la inyección intracordal, tiroplastía tipo I, aducción aritenoidea y reinervaciones laríngeas. Esta última ofrece la ventaja de prevenir la pérdida progresiva del tono muscular y el volumen del músculo tiroaritenoide observado con la denervación de las cuerdas vocales ${ }^{24}$. Dada la falta de experiencia en reinervación del nervio laríngeo recurrente en nuestro centro, se propone una eventual tiroplastía posterior.

En caso de que la longitud de la porción resecada exceda los $6 \mathrm{~cm}$, es necesario realizar una liberación suprahioidea, mediante la sección de los músculos infrahiodeos a nivel del hioides o torácica mediante la sección del ligamento triangular pulmonar, lo que otorga la posibilidad de aumentar unos centímetros ${ }^{16}$. Dado que es un procedimiento complejo, con una morbilidad global de $26 \%$ y una mortalidad de $1,2 \%{ }^{25}$, requiere de equipos quirúrgicos especializados. Se debe considerar que ni la radioterapia ni el radioyodo compensarán cirugías incompletas.

En cuanto al estudio histológico, en ambos casos se informó un carcinoma papilar de tiroides con infiltración transmural de la pared traqueal sin especificar un subtipo predominante, entendiendo la heterogeneidad histológica que no es infrecuente en este tipo de tumores. Es necesario recalcar, que ambos casos llevan un periodo corto desde su cirugía, no siendo posible aún evaluar el control locorregional a largo plazo.

Finalmente, el radioyodo posquirúrgico estará indicado en todos los tumores diferenciados captantes y la radioterapia en caso de márgenes positivos o cercanos, tumores pobremente diferenciados, múltiples recurrencias e invasión de estructuras mayores. Por otro lado, en caso de malas condiciones generales, extensión locorregionales múltiples o alta carga de metástasis a distancia, pudiese ser apropiado realizar tratamientos quirúrgicos más conservadores, incluyendo shaving más radioterapia posoperatoria ${ }^{20}$.

\section{Conclusión}

Si bien el cáncer de tiroides en términos generales es de buen pronóstico, la extensión extratiroidea es un factor importante a evaluar que incide directamente en el manejo y supervivencia. La evaluación preoperatoria en conjunto con el conocimiento de las alternativas terapéuticas son esenciales para ofrecer un apropiado manejo a estos pacientes.

\section{Bibliografía}

1. Trigo JM, Capdevila J, Grande E, Grau J, Lianes P, Spanish Society for Medical Oncology. Thyroid cancer: SEOM clinical guidelines. Clin Transl Oncol. 2014;16:1035-1042.

2. Howlander N, Noone AM, Krapcho M, et al. SEER Cancer Statistics Review, 1975-2013. Bethesda: National Cancer Institute, 2016.

3. Shaha AR, Ferlito A, Owen RP, Silver CE, Rodrigo JP, Haigentz M Jr, Mendenhall WM, et al. Airway issues in anaplastic thyroid carcinoma. Eur Arch Otorhinolaryngol. 2013;270(10):2579-2583.

4. Shah JP, Loree TR, Dharker D, et al. Prognostic factors in differentiated carcinoma of the thyroid gland. Am J Surg. 1992;164(6):658-661.

5. Andersen PE, Kinsella J, Loree TR, et al. Differentiated carcinoma of the thyroid with extrathyroidal extension. Am J Surg. 1995;170(5):467470.

6. Hay ID, McConahey WM, Goellner JR. Managing patients with papillary thyroid carcinoma: insights gained from the Mayo Clinic's experience of treating 2512 consecutive patients during 1940 through 2000. Trans Am Clin Climatol Assoc. 2002;113:241-260.

7. McCaffrey TV, Bergstralh EJ, Hay ID. Locally invasive papillary thyroid carcinoma: 1940-1990. Head Neck. 1994;16(2):165-172.

8. Brauckhoff M. Classification of aerodigestive tract invasion from thyroid cancer. Langenbecks Arch Surg. 2014;399(2):209-216. doi: 10.1007/s00423-0131142-x. 


\section{CASO CLÍNICO}

9. Randolph, Gregory W. Surgery of the thyroid and parathyroid glands: expert consult premium editionenhanced online features and print. Elsevier Health Sciences, 2012.

10. Ishihara T, Yamazaki S, Kobayashi K, et al. Resection of the trachea infiltrated by thyroid carcinoma. Ann Surg. 1982;195:496-500.

11. McCaffrey TV, Lipton RJ. Thyroid carcinoma invading the upper aerodigestive system. Laryngoscope. 1990;100(8):824-830.

12. Ishigaki S, Shimamoto K, Satake H, et al. Multislice CT of thyroid nodules: comparison with ultrasonography. Radiat Med. 2004;22(5):346-353.

13. Chung SR, Baek JH, Choi YJ, et al. The Role of Core Needle Biopsy for the Evaluation of Thyroid Nodules with Suspicious Ultrasound Features. Korean J Radiol. 2019;20(1):158-165. doi: 10.3348/ kjr.2018.0101.

14. Chmielik E, Rusinek D, Oczko-Wojciechowska M, Jarzab M, Krajewska J, Czarniecka A, Jarzab B: Heterogeneity of Thyroid Cancer. Pathobiology. 2018;85:117-129. doi: 10.1159/000486422.

15. Brierley JD, Gospodarowicz MK, Wittekind C. TNM Classification of Malignant Tumours. 8 th ed. UICC; John Wiley \& Sons, Ltd. 2017.

16. Shin DH, Mark EJ, Suen HC, Grillo HC. Pathologic staging of papillary carcinoma of the thyroid with airway invasion based on the anatomic manner of extension to the trachea: a clinicopathologic study based on 22 patients who underwent thyroidectomy and airway resection. Hum Pathol. 1993;24(8):866870. doi: 10.1016/0046-8177(93)90136-5.

17. Czaja JM, McCaffrey TV. The surgical management of laryngotracheal invasion by well-differentiated papillary thyroid carcinoma. Arch Otolaryngol Head Neck Surg. 1997;123(5):484-490. doi: 10.1001/ archotol.1997.01900050030003.

18. Price DL, Wong RJ, Randolph GW. Invasive thyroid cancer: management of the trachea and esophagus. Otolaryngol Clin North Am. 2008;41(6):1155-x. doi: 10.1016/j.otc.2008.08.002.

19. Honings J, Stephen A, Marres H, Gaissert H. The Management of Thyroid Carcinoma Invading the Larynx or Trachea. Laryngoscope. 2010; 120:682-689.

20. Nishida T, Nakao K, Hamaji M. Differentiated thyroid carcinoma with airway invasion: indication for tracheal resection based on the extent of cancer invasion. J Thorac Cardiovasc Surg. 1997;114(1):8492.

21. Xu J, Liao Z, Li JJ, Wu XF, Zhuang SM. The Role of Tracheostomy in Anaplastic Thyroid Carcinoma. World J Oncol. 2015;6(1):262-264. doi: 10.14740/ wjon 899 w.

22. Grillo HC. Circumferential resection and reconstruction of the mediastinal and cervical trachea. Ann Surg. 1965;162:374-388.

23. Suárez C, Gil-Carcedo L, Marco J, Medina J, Ortega P, Trinidad J. Tratado de Otorrinolaringología y Cirugía de Cabeza y Cuello. 2a Edición, Tomo IV, 2008;238:3619-3624.

24. Sanuki T, Yumoto E, Minoda R, Kodama N. The role of immediate recurrent laryngeal nerve reconstruction for thyroid cancer surgery. J Oncol. 2010;2010:846235. doi: 10.1155/2010/846235.

25. Gaissert HA, Honings J, Grillo HC, et al. Segmental laryngotracheal and tracheal resection for invasive thyroid carcinoma. Ann Thorac Surg. 2007;83:195259. 